\title{
Matchings on infinite graphs
}

\author{
Charles Bordenave * Marc Lelarge ${ }^{\dagger}$ and Justin Salez ${ }^{\ddagger}$
}

\begin{abstract}
Elek and Lippner (2010) showed that the convergence of a sequence of bounded-degree graphs implies the existence of a limit for the proportion of vertices covered by a maximum matching. We provide a characterization of the limiting parameter via a local recursion defined directly on the limit of the graph sequence. Interestingly, the recursion may admit multiple solutions, implying non-trivial long-range dependencies between the covered vertices. We overcome this lack of correlation decay by introducing a perturbative parameter (temperature), which we let progressively go to zero. This allows us to uniquely identify the correct solution. In the important case where the graph limit is a unimodular Galton-Watson tree, the recursion simplifies into a distributional equation that can be solved explicitly, leading to a new asymptotic formula that considerably extends the well-known one by Karp and Sipser for Erdős-Rényi random graphs.
\end{abstract}

\section{Introduction}

A matching on a finite graph $G=(V, E)$ is a subset of pairwise non-adjacent edges $M \subseteq E$. The $|V|-2|M|$ isolated vertices of $(V, M)$ are said to be exposed by $M$. We let $\mathbb{M}(G)$ denote the set of all possible matchings on $G$. The matching number of $G$ is defined as

$$
\nu(G)=\max _{M \in \mathbb{M}(G)}|M|
$$

and those $M$ which achieve this maximum - or equivalently, have the fewest exposed vertices are called maximum matchings. The normalized matching number of $G$ is simply $\nu(G) /|V|$.

Our results belong to the theory of convergent graph sequences. Convergence of bounded degree graph sequences was defined by Benjamini and Schramm [7], Aldous and Steele [2], see also Aldous and Lyons [1]. The notion of local weak convergence has then inspired a lot of work [8], [11], 13], 16], 25]. In [17], it is shown that the convergence of a sequence of bounded-degree graphs guarantees the existence of a limit for their normalized matching numbers. We provide a characterization of the limiting parameter via a local recursion defined directly on the limit of the graph sequence. In the important case where the graph limit is a unimodular Galton-Watson tree, the recursion simplifies into a distributional equation that can be solved explicitly, leading to a new asymptotic formula.

\footnotetext{
${ }^{*}$ Institut de Mathématiques - Univ. de Toulouse \& CNRS - France. Email: bordenave@math.univ-toulouse.fr

${ }^{\dagger}$ INRIA-École Normale Supérieure - France. Email: marc.lelarge@ens.fr

${ }^{\ddagger}$ INRIA-École Normale Supérieure - France. Email: justin.salez@ens.fr
} 
A classical example in this context is the Erdős-Rényi random graph with average degree $c$ on $n$ vertices, denoted by $G(n, c / n)$ : as $n \rightarrow \infty, G(n, c / n)$ converges in the local weak sense to a Galton-Watson tree with degree distribution Poisson with parameter $c$. In this case, Karp and Sipser [22] showed that

$$
\frac{\nu(G(n, c / n))}{n} \underset{n \rightarrow \infty}{\longrightarrow} 1-\frac{t_{c}+e^{-c t_{c}}+c t_{c} e^{-c t_{c}}}{2}
$$

where $t_{c} \in(0,1)$ is the smallest root of $t=e^{-c e^{-c t}}$ (we will see in the sequel that the convergence is almost sure). The explicit formula (2) rests on the analysis of a heuristic algorithm now called Karp-Sipser algorithm. The latter is based on the following observation : if $e \in E$ is a pendant edge (i.e. an edge incident to a vertex of degree one) in $G=(V, E)$, then there is always a maximum matching that contains $e$, so all edges that are adjacent to $e$ may be deleted without affecting $\nu(G)$. The first stage of the algorithm consists in iterating this until no more pendant edge is present. This is the leaf-removal process. $G$ is thus simplified into a sub-graph with only isolated vertices, matched pairs, and a so-called core with minimum degree at least 2 . As long as that core is non-empty, one of its edges is selected uniformly at random, the adjacent edges are deleted, and the whole process starts again. When the algorithm stops, the remaining edges clearly form a matching on $G$, but its size may be far below $\nu(G)$ due to the sub-optimal removals on the core.

On $G(n, c / n)$, the dynamics of the deletion process can be approximated in the $n \rightarrow \infty$ limit by differential equations which can be explicitly solved. In particular, the asymptotic size of both the optimal part constructed in the first stage, and the sub-optimal part constructed on the core can be evaluated up to an $o(n)$ correcting term (which has been later refined, see [4]). Moreover, the second part happens to be almost perfect, in the sense that only $o(n)$ vertices are exposed in the core. This guarantees that the overall construction is asymptotically optimal, and the asymptotic formula for $\nu\left(G_{n}\right)$ follows. More recently, the same technique has been applied to another class of random graphs with a fixed log-concave degree profile [10, resulting in the asymptotical existence of an almost perfect matching on these graphs :

$$
\frac{\nu\left(G_{n}\right)}{\left|V_{n}\right|} \underset{n \rightarrow \infty}{\longrightarrow} \frac{1}{2}
$$

In both cases, the proof of optimality - and hence the asymptotic formula for $\nu\left(G_{n}\right)$ - relies on the fact that the second stage exposes only $o(n)$ vertices, which is bound to fail as soon as one considers more general graph ensembles where the core does not necessarily admits an almostperfect matching. We give simple examples in the Appendix. By using a completely different approach - namely establishing and solving an appropriate recursive distributional equation (a usual ingredient of the objective method, see [3] - , we manage to obtain a general formula that considerably extends the above results.

The rest of our paper is organized as follows: we state our main results in Section 2, In Section 3, we extend the Boltzmann-Gibbs distribution over matchings on a finite graph to infinite graphs. This will allow us to derive our Theorem 1 in Section 4. We deal with the specific cases of trees (and random graphs) in Section 5. We end the paper with an Appendix presenting simple examples of graphs for which the limiting local recursion admit multiple solutions. 


\section{Results}

Let us start with a brief recall on local weak convergence (see [7, 2] for details). A rooted graph $(G, \circ)$ is a graph $G=(V, E)$ together with the specification of a particular vertex $\circ \in V$, called the root. We let $\mathcal{G}$ denote the set of all locally finite connected rooted graphs considered up to rooted isomorphism, i.e. $\left(G_{1}, \circ_{1}\right) \equiv\left(G_{2}, \circ_{2}\right)$ if there exists a bijection $\gamma: V_{1} \rightarrow V_{2}$ that preserves roots $\left(\gamma\left(\circ_{1}\right)=\circ_{2}\right)$ and adjacency $\left(u v \in E_{1} \Longleftrightarrow \gamma(u) \gamma(v) \in E_{2}\right)$. In the space $\mathcal{G}$, a sequence $\left\{\left(G_{n}, \circ_{n}\right) ; n \in \mathbb{N}\right\}$ converges locally to $(G, \circ)$ if for every radius $k \in \mathbb{N}$, there is $n_{k} \in \mathbb{N}$ such that

$$
n \geq n_{k} \Longrightarrow\left[G_{n}, \circ_{n}\right]_{k} \equiv[G, \circ]_{k} .
$$

Here, $[G, \circ]_{k}$ denotes the finite rooted subgraph induced by the vertices lying at graph-distance at most $k$ from $\circ$. It is not hard to construct a distance which metrizes this notion of convergence and turns $\mathcal{G}$ into a complete separable metric space. We can thus import the usual machinery of weak convergence of probability measures on Polish spaces (see e.g. [9]). We define $\mathcal{P}(G)$ as the set of probability measure on $\mathcal{G}$. There is a natural procedure for turning a finite deterministic graph $G=(V, E)$ into a random element of $\mathcal{G}$ : one simply chooses uniformly at random a vertex $\circ \in V$ to be the root, and then restrains $G$ to the connected component of $\circ$. The resulting law is denoted by $\mathcal{U}(G) \in \mathcal{P}(\mathcal{G})$. If $\left(G_{n}\right)_{n \in \mathbb{N}}$ is a sequence of finite graphs such that $\left(\mathcal{U}\left(G_{n}\right)\right)_{n \in \mathbb{N}}$ admits a weak limit $\rho \in \mathcal{P}(\mathcal{G})$, we call $\rho$ the random weak limit of the sequence $\left(G_{n}\right)_{n \in \mathbb{N}}$. Finally, for any $d \geq 0$, we define $\mathcal{G}_{d}$ as the space of all rooted connected graphs with maximal degree no more than $d$.

Rather than just graphs $G=(V, E)$, it will be sometimes convenient to work with discrete networks $G=(V, E, \mathcal{M})$, in which the additional specification of a mark map $\mathcal{M}: E \rightarrow \mathbb{N}$ allows to attach useful local information to edges, such as their absence/presence in a certain matching. We then simply require the isomorphisms in the above definition to preserve these marks.

The first main implication of our work is that the local weak convergence of a sequence of graphs is enough to guarantee the convergence of their normalized matching numbers to a quantity that can be described directly on the random weak limit of the graph sequence.

Theorem 1 Let $G_{n}=\left(V_{n}, E_{n}\right), n \in \mathbb{N}$, be a sequence of finite graphs admitting a random weak limit $\rho$. Then,

$$
\frac{\nu\left(G_{n}\right)}{\left|V_{n}\right|} \underset{n \rightarrow \infty}{\longrightarrow} \gamma
$$

where $\gamma \in\left[0, \frac{1}{2}\right]$ is defined by a recursion defined directly on the random weak limit $\rho$.

Since the work of Heilmann and Lieb [21], it is known that the thermodynamic limit for monomer-dimer systems exists and basic properties of this limit are derived for lattices. In particular, [21, Lemma 8.7] shows the convergence of the normalized matching number when the underlying graph is a lattice. More recently, Elek and Lippner [17] extended this result by using the framework of local weak convergence for bounded degree graphs. Here we remove the bounded degree assumption. More importantly, the approach in [17] is non-constructive. In contrast, we provide a characterization of $\gamma$ in terms of a local recursion defined directly on the random weak limit $\rho$. We postpone the discussion on how to actually compute $\gamma$ from $\rho$ to Subsection [3.1. Our approach starts as in [21] with the introduction of a natural family of probability distributions on the set of matchings parametrized by a single parameter $z>0$ 
called the Boltzmann-Gibbs distribution. The analysis in [21] concentrates on the properties of the partition function, also known as the matching polynomial, from which a result like (4) can be deduced. Our analysis differs from this approach and concentrates on the analysis of the local marginals of the Boltzmann-Gibbs distribution, in a similar spirit as in the (non-rigorous) work of Zdeborová and Mézard [26]. As in [26] and [20], we start from an elementary formal recursion satisfied by the matching polynomials, and deduce an exact recursion for the local marginals of the Boltzmann-Gibbs distribution on any finite graph. A careful analysis of the contractivity properties of this recursion allows us to define the monomer-dimer model on infinite graphs (see Theorem 6), and to define their "normalized matching number" (see Theorem 10). We should stress that the analysis of the marginal probabilities is essential for our second main result, namely the explicit computation of the matching number when the local weak limit is a Galton-Watson tree. Although simple adaptations of the argument in [21] would yield a result like Theorem 1, the limit would be given in an implicit way which would not be sufficient to get our second main result.

As many other classical graph sequences, Erdős-Rényi graphs and random graphs with a prescribed degree profile admit almost surely a particularly simple random weak limit, namely a unimodular Galton-Watson (UGW) tree (see Example 1.1 in [1]). This random rooted tree is parametrized by a probability distribution $\pi \in \mathcal{P}(\mathbb{N})$ with finite mean, called its degree distribution. It is obtained by a Galton-Watson branching process where the root has offspring distribution $\pi$ and all other genitors have offspring distribution $\widehat{\pi} \in \mathcal{P}(\mathbb{N})$ defined by

$$
\forall n \in \mathbb{N}, \widehat{\pi}_{n}=\frac{(n+1) \pi_{n+1}}{\sum_{k} k \pi_{k}}
$$

Thanks to the markovian nature of the branching process, the recursion defining $\gamma$ simplifies into a recursive distributional equation, which has been explicitly solved by the authors in a different context [12].

Theorem 2 With the notation of Theorem 1, if the random weak limit $\rho$ is a UGW tree with degree distribution $\pi$, we have the explicit formula

$$
\gamma=\frac{1-\max _{t \in[0,1]} F(t)}{2}
$$

where

$$
F(t)=t \phi^{\prime}(1-t)+\phi(1-t)+\phi\left(1-\frac{\phi^{\prime}(1-t)}{\phi^{\prime}(1)}\right)-1
$$

and $\phi(t)=\sum_{n} \pi_{n} t^{n}$ is the moment generating function of the degree distribution $\pi$.

Differentiating the above expression, we see that any $t$ achieving the maximum must satisfy

$$
\phi^{\prime}(1) t=\phi^{\prime}\left(1-\frac{\phi^{\prime}(1-t)}{\phi^{\prime}(1)}\right) .
$$

For Erdös-Rényi random graphs with connectivity $c$, the degree of the limiting UGW tree is Poisson with parameter $c$ (i.e. $\phi(t)=\exp (c t-c)$ ), so that (6) becomes $t=e^{-c e^{-c t}}$. We thus recover precisely Karp and Sipser's formula (2). Similarly, for random graphs with a prescribed 
degree sequence, the log-concave assumption made by Bohmann and Frieze guarantees that the above maximum is achieved at $t=0$ with $F(0)=0$, hence (3) follows automatically.

A classical area of combinatorial optimization is formed by bipartite matching [24. We end this section, with a specialization of our results to bipartite graphs $G=\left(V=V^{a} \cup V^{b}, E\right)$. The natural limit for a sequence of bipartite graphs is the following hierarchal Galton-Watson tree parameterized by two distributions on $\mathbb{N}$ with finite first moment, $\pi^{a}$ and $\pi^{b}$ and a parameter $\lambda \in[0,1]$. We denote $\widehat{\pi}^{a}$ and $\widehat{\pi}^{b}$ the corresponding distributions given by the transformation (5). We also denote $\phi^{a}$ and $\phi^{b}$ the generating functions of $\pi^{a}$ and $\pi^{b}$. The hierarchal Galton-Watson tree is then defined as follows: with probability $\lambda$, the root has offspring distribution $\pi^{a}$, all odd generation genitors have offspring distribution $\widehat{\pi}^{b}$ and all even generation genitors have offspring distribution $\widehat{\pi}^{a}$; similarly with probability $1-\lambda$, the root has offspring distribution $\pi^{b}$, all odd generation genitors have offspring distribution $\widehat{\pi}^{a}$ and all even generation genitors have offspring distribution $\widehat{\pi}^{b}$. In the first (resp. second) case, we say that the root and all even generations are of type $a$ (resp. $b$ ) and all the odd generations are of type $b$ (resp. $a$ ). To get a unimodular hierarchal Galton-Watson ( $U H G W$ ) tree with degree distributions $\pi^{a}$ and $\pi^{b}$, we need to have: $\lambda \phi^{a \prime}(1)=(1-\lambda) \phi^{b \prime}(1)$, so that

$$
\lambda=\frac{\phi^{b \prime}(1)}{\phi^{a \prime}(1)+\phi^{b \prime}(1)} .
$$

Theorem 3 With the notation of Theorem 1, assume that the random weak limit $\rho$ is a $U H G W$ tree with degree distributions $\pi^{a}, \pi^{b}$. If $\pi^{a}$ and $\pi^{b}$ have finite first moment, then

$$
\gamma=\frac{\phi^{b \prime}(1)}{\phi^{a \prime}(1)+\phi^{b \prime}(1)}\left(1-\max _{t \in[0,1]} F^{a}(t)\right)
$$

where $F^{a}$ is defined by:

$$
F^{a}(t)=\phi^{a}\left(1-\frac{\phi^{b \prime}(1-t)}{\phi^{b \prime}(1)}\right)-\frac{\phi^{a \prime}(1)}{\phi^{b \prime}(1)}\left(1-\phi^{b}(1-t)-t \phi^{b \prime}(1-t)\right) .
$$

Note that if $\phi^{a}(x)=\phi^{b}(x)$, we find the same limit as in Theorem 2. Note that it is not obvious from formula (8) that our expression for $\gamma$ is symmetric in $a$ and $b$ as it should. In the forthcoming Section 5.2. Equation (30) gives an alternative symmetric formula for $\gamma$ which simplifies to (8) thanks to (7).

Note also that our Theorem 3 computes the independence number of random bipartite graphs. Recall that a set of vertices in a graph $G$ is said to be independent if no two of them are adjacent. The cardinality of any largest independent set of points in $G$ is known as the independence number of $G$ or the stability number of $G$ and is denoted by $\alpha(G)$. By König's theorem, we know that for any bipartite graph $G$ with vertex set $V, \alpha(G)+\nu(G)=|V|$. The fact that a limit for $\frac{\alpha\left(G_{n}\right)}{\left|V_{n}\right|}$ exists, has been proved recently in [5] for Erdős-Rényi and random regular graphs. The actual value for this limit is unknown except for Erdös-Rényi graphs with mean degree $c<e$. In this case, the leaf-removal algorithm allows to compute explicitly the limit which agrees with (8) with $\phi^{a}(x)=\phi^{b}(x)=\exp (c x-x)$.

Motivated by some applications for Cuckoo Hashing [18, [15], recent results have been obtained in the particular case where $\pi^{a}(k)=1$ for some $k \geq 3$ and $\pi^{b}$ is a Poisson distribution 
with parameter $\alpha k$. These degree distributions arise if one consider a sequence of bipartite graphs with $\lfloor\alpha m\rfloor$ nodes of type $a$ (called the items), $m$ nodes of type $b$ (called the locations) and each node of type $a$ is connected with $k$ nodes of type $b$ chosen uniformly at random (corresponding to the assigned locations the item can be stored in). The result in this domain, obtained in [19] follows (see Section 5.3) from our Theorem 3, namely:

Corollary 4 Under the assumption of Theorem 3 and with $\pi^{a}(k)=1$ for some $k \geq 3$ and $\pi^{b}$ is a Poisson distribution with parameter $\alpha k$. Let $\xi$ be the unique solution of the equation:

$$
k=\frac{\xi\left(1-e^{-\xi}\right)}{1-e^{-\xi}-\xi e^{-\xi}}
$$

and $\alpha_{c}=\frac{\xi}{k\left(1-e^{-\xi}\right)^{k-1}}$.

- for $\alpha \leq \alpha_{c}$, all (except $\left.o_{p}(n)\right)$ vertices of type a are covered, i.e. $\frac{\nu\left(G_{n}\right)}{\left|V_{n}^{a}\right|} \underset{n \rightarrow \infty}{\longrightarrow} 1$.

- for $\alpha>\alpha_{c}$, we have:

$$
\frac{\nu\left(G_{n}\right)}{\left|V_{n}^{a}\right|} \underset{n \rightarrow \infty}{\longrightarrow} 1-\frac{1}{\alpha}\left(e^{-\xi^{*}}+\xi^{*} e^{-\xi^{*}}+\frac{\xi^{*}}{k}\left(1-e^{-\xi^{*}}\right)-1\right),
$$

where $\xi^{*}=k \alpha x^{*}$ and $x^{*}$ is the largest solution of $x=\left(1-e^{-k \alpha x}\right)^{k-1}$.

In words, $\alpha_{c}$ is the load threshold: if $\alpha \leq \alpha_{c}$, there is an assignment of the $\lfloor\alpha m\rfloor$ items to a table with $m$ locations that respects the choices of all items, whereas for $\alpha>\alpha_{c}$, such an assignement does not exist and (9) gives the maximal number of items assigned without collision. Note that results in [18, [15] are slightly different in the sense that for the specific sequence of random graphs described above (i.e. uniform hypergraphs), they show that for $\alpha<\alpha_{c}$ all vertices of type $a$ are covered with high probability. It is shown in [23] how to get such results from Corollary 4 under the additional assumption that the sequence of graphs are uniform hypergraphs.

\section{The Monomer-Dimer model}

We start with the case of a finite graph $G=(V, E)$. Consider a natural family of probability distributions on the set of matchings $\mathbb{M}(G)$, parameterized by a single parameter $z>0$ called the temperature (note that the standard temperature $T$ in physics would correspond to $z=e^{-1 / T}$ but this will not be important here): for any $M \in \mathbb{M}(G)$,

$$
\mu_{G}^{z}(M)=\frac{z^{|V|-2|M|}}{P_{G}(z)}
$$

where $P_{G}$ is the matching polynomial, $P_{G}(z)=\sum_{M \in \mathbb{M}(G)} z^{|V|-2|M|}$. In statistical physics, this is called the monomer-dimer model at temperature $z$ on $G$ (see [21] for a complete treatment). We let $\mathcal{M}_{G}^{z}$ denote a random element of $\mathbb{M}(G)$ with law $\mu_{G}^{z}$, and we call it a Boltzmann random matching at temperature $z$ on $G$. Note that the lowest degree coefficient of $P_{G}$ is precisely the number of largest matchings on $G$. Therefore, $\mathcal{M}_{G}^{z}$ converges in law to a uniform largest 
matching as the temperature $z$ tends to zero. We define the root-exposure probability (REP) of the rooted graph $(G, \circ)$ as

$$
\mathcal{R}_{z}[G, \circ]=\mu_{G}^{z}(\circ \text { is exposed })
$$

\subsection{Local recursions to compute $\gamma$}

Before starting with the proof, we explain (whithout proofs) how to compute $\gamma$ in (44). For a finite graph, our computation of $\gamma$ follows exactly the approach of Godsil [20. We recall Godsil's notion of the path-tree associated with a rooted graph $G$ : if $G$ is any rooted graph with root $\circ$, we define its path-tree $T_{G}$ as the rooted tree whose vertex-set consists of all finite simple paths starting at the root $\circ$; whose edges are the pairs $\left\{P, P^{\prime}\right\}$ of the form $P=v_{1} \ldots v_{n}$, $P^{\prime}=v_{1} \ldots v_{n} v_{n+1}(n \geq 1)$; whose root is the single-vertex path $\circ$. By a finite simple path, we mean here a finite sequence of distinct vertices $v_{1} \ldots v_{n}(n \geq 1)$ such that $v_{i} v_{i+1} \in E$ for all $1 \leq i<n$.

It is well-known since Godsil's result [20] that path-trees capture considerable information about matchings in general graph and are easier to work with than the graph itself. For a rooted graph $[G, \circ]$, let $T_{[G, \circ]}$ be the associated path-tree and consider the corresponding system of equations (where $u \succ v$ if $u$ is a child of $v$ ):

$$
\forall v \in T_{[G, \circ]}, \quad x_{v}=\frac{1}{1+\sum_{u \succ v}\left(\sum_{w \succ u} x_{w}\right)^{-1}} .
$$

For any finite rooted graph $[G, \circ],(\underline{12})$ has a unique solution in $[0,1]^{T_{[G, \circ]}}$ and we denote the value taken at the root by $x_{\circ}(G)$. Then $x_{\circ}(G)$ is exactly the probability for the root $\circ$ of being exposed in a uniform maximal matching. In particular, we have

$$
\nu(G)=\sum_{v \in V} \frac{1-x_{v}(G)}{2} .
$$

This argument follows from [20] and will be a special case of our analysis.

For an infinite graph with bounded degree, it turns out that it is not always possible to make sense of the local recursions (12). However, our analysis will show that for any $z>0$, the infinite set of equations:

$$
\forall v \in T_{[G, \circ]}, \quad x_{v}(z)=\frac{1}{1+\sum_{u \succ v}\left(z^{2}+\sum_{w \succ u} x_{w}(z)\right)^{-1}},
$$

has a unique solution in $[0,1]^{T[G, \circ]}$ and the value taken by the root is exactly $\mathcal{R}_{z}[G, \circ]$ (which is the probability for the root $\circ$ of being exposed in a Boltzmann random matching at temperature $z$ when the graph $G$ is finite). Then our Theorem 11 will imply that for any sequence of finite graphs $\left(G_{n}=\left(V_{n}, E_{n}\right)\right)_{n \in \mathbb{N}}$ satisfying $\left|E_{n}\right|=O\left(\left|V_{n}\right|\right)$ and having $\rho$ as a random weak limit,

$$
\frac{\nu\left(G_{n}\right)}{\left|V_{n}\right|} \underset{n \rightarrow \infty}{\longrightarrow} \frac{1-\mathbb{E}_{\rho}\left[\lim _{z \rightarrow 0} \mathcal{R}_{z}\right]}{2}
$$

and $\lim _{z \rightarrow 0} \mathcal{R}_{z}[G, \circ]$ is actually the largest solution to (12). From a practical point of view, it is possible to compute an approximation of $\mathcal{R}_{z}[G, \circ]$ by looking at a sufficient large ball centered at the root $\circ$. Moreover our analysis will show that the quantity $\mathbb{E}_{\rho}\left[\mathcal{R}_{z}\right]$ is a good approximation of $\mathbb{E}_{\rho}\left[\lim _{z \rightarrow 0} \mathcal{R}_{z}\right]$ as soon as $\left|E_{n}\right|=O\left(\left|V_{n}\right|\right)$ (see Lemma 12). 


\subsection{Extension of the model on infinite graphs with bounded degree}

Let $G$ - $\circ$ be the graph obtained from $G$ by removing its root $\circ$. Since the matchings of $G$ that expose $\circ$ are exactly the matchings of $G-\circ$, we have the identity

$$
\mathcal{R}_{z}[G, \circ]=\frac{z P_{G-\circ}(z)}{P_{G}(z)},
$$

which already shows that the REP is an analytic function of the temperature. The remarkable fact that its domain of analyticity contains the right complex half-plane

$$
\mathbb{H}_{+}=\{z \in \mathbb{C} ; \Re(z)>0\}
$$

is a consequence of the powerful Heilmann-Lieb theorem [21, Theorem 4.2] (see [14] for generalizations). The key to the study of the REP is the following elementary but fundamental local recursion :

$$
\mathcal{R}_{z}[G, \circ]=z^{2}\left(z^{2}+\sum_{v \sim \circ} \mathcal{R}_{z}[G-\circ, v]\right)^{-1} .
$$

Clearly, this recursion determines uniquely the functional $\mathcal{R}_{z}$ on the class of finite rooted graphs, and may thus be viewed as an inductive definition of the REP. Remarkably enough, this alternative characterization allows for a continuous extension to infinite graphs with bounded degree, even though the above recursion never ends. We let $\mathcal{H}$ denote the space of analytic functions on $\mathbb{H}_{+}$, equipped with its usual topology of uniform convergence on compact sets. Our fundamental lemma is as follows:

\section{Theorem 5 (The fundamental local lemma)}

1. For every fixed $z \in \mathbb{H}_{+}$, the local recursion (14) determines a unique $\mathcal{R}_{z}: \mathcal{G}_{d} \rightarrow z \mathbb{H}_{+}$.

2. For every fixed $[G, \circ] \in \mathcal{G}_{d}, z \mapsto \mathcal{R}_{z}[G, \circ]$ is analytic.

3. The resulting mapping $[G, \circ] \in \mathcal{G}_{d} \longmapsto \mathcal{R}_{(\cdot)}[G, \circ] \in \mathcal{H}$ is continuous.

This local lemma has strong implications for the monomer-dimer model, which we now list. The first one is the existence of an infinite volume limit for the Gibbs-Boltzmann distribution.

Theorem 6 (Monomer-dimer model on infinite graphs) Consider a graph $G \in \mathcal{G}_{d}$ and a temperature $z>0$. For any finite matching $M$ of $G$, the cylinder-event marginals defined by

$$
\mu_{G}^{z}(M \subseteq \mathcal{M})=z^{-2|M|} \prod_{k=1}^{2|M|} \mathcal{R}_{z}\left[G-\left\{v_{1}, \ldots, v_{k-1}\right\}, v_{k}\right],
$$

are consistent and independent of the ordering $v_{1}, \ldots v_{2|M|}$ of the vertices spanned by $M$. They thus determine a unique probability distribution $\mu_{G}^{z}$ over the matchings of $G$. It coincides with the former definition in the case where $G$ is finite, and extends it continuously in the following sense : for any $\circ \in V$ and any sequence $\left(\left[G_{n}, \circ_{n}\right]\right)_{n \in \mathbb{N}} \in \mathcal{G}_{d}{ }^{\mathbb{N}}$ converging to $[G, \circ]$,

$$
\left[G_{n}, \circ_{n}, \mathcal{M}_{n}\right] \underset{n \rightarrow \infty}{\stackrel{d}{\longrightarrow}}[G, \circ, \mathcal{M}]
$$

in the local weak sense for random networks, where $\mathcal{M}_{n}$ has law $\mu_{G_{n}}^{z}$ and $\mathcal{M}$ has law $\mu_{G}^{z}$. 
Although it is not our concern here, we obtain as a by-product the strong convergence of the logarithm of the matching polynomial, also called free energy in the monomer-dimer model :

Corollary 7 Let $\left(G_{n}\right)_{n \in \mathbb{N}}$ be a sequence of finite graphs with bounded degree admitting a random weak limit $[G, \circ]$. The following convergence holds in the analytic sense on $\mathbb{H}_{+}$,

$$
\frac{1}{\left|V_{n}\right|} \log \frac{P_{G_{n}}(z)}{P_{G_{n}}(1)} \underset{n \rightarrow \infty}{\longrightarrow} \int_{1}^{z} \frac{\mathbb{E}_{\rho}\left[\mathcal{R}_{s}[G, \circ]\right]}{s} d s
$$

where $\mathbb{E}_{\rho}\left[\mathcal{R}_{s}[G, \circ]\right]$ denotes the expectation under the measure $\rho$ of the variable $\mathcal{R}_{s}[G, \circ]$.

A similar result was established in [21] for the lattice case, and in [6] under a restrictive large girth assumption.

\subsection{Proof of Theorem [5: the fundamental lemma}

The local recursion (14) involves mappings of the form :

$$
\phi_{z, d}:\left(x_{1}, \ldots, x_{d}\right) \mapsto z^{2}\left(z^{2}+\sum_{i=1}^{d} x_{i}\right)^{-1}
$$

where $d \in \mathbb{N}$. In the following lemma, we gather a few elementary properties of this transformation, which are immediate to check but will be of constant use throughout the paper.

Lemma 8 (Elementary properties) For any $d \in \mathbb{N}$ and $z \in \mathbb{H}_{+}$,

1. $\phi_{z, d}$ maps analytically $z \mathbb{H}_{+} \times \ldots \times z \mathbb{H}_{+}$into $z \mathbb{H}_{+}$

2. $\left|\phi_{z, d}\right|$ is uniformly bounded by $|z| / \Re(z)$ on $z \mathbb{H}_{+} \times \ldots \times z \mathbb{H}_{+}$.

From part 1, it follows that the REP of a finite rooted graph belongs to $\mathcal{H}$, when viewed as a function of the temperature $z$. Part 2 and Montel's theorem guarantee that the family of all those REPs is relatively compact in $\mathcal{H}$. Note that relative compactness also plays a central role in [21. Combined with the following uniqueness property at high temperature, it will quickly lead to the proof of Theorem 5 .

The local recursion (14) also involves graph transformations of the form $[G, \circ] \mapsto[G-$ $\circ, v]$, where $v \sim \circ$. Starting from a given $[G, \circ] \in \mathcal{G}_{d}$, we let $\operatorname{Succ}^{*}[\mathrm{G}, \circ] \subseteq \mathcal{G}_{\mathrm{d}}$ denote the (denumerable) set of all rooted graphs that can be obtained by successively applying finitely many such transformations.

Lemma 9 (Uniqueness at high temperature) Let $[G, \circ] \in \mathcal{G}_{d}$ and $z \in \mathbb{H}_{+}$such that $\Re(z)>$ $\sqrt{d}$. If

$$
\mathcal{R}_{z}^{1}, \mathcal{R}_{z}^{2}: \operatorname{Succ}^{*}[\mathrm{G}, \circ] \rightarrow \mathrm{z} \mathbb{H}_{+}
$$

both satisfy the local recursion (14) then $\mathcal{R}_{z}^{1}=\mathcal{R}_{z}^{2}$. 
Proof. Set $\alpha=2|z| / \Re(z)$ and $\beta=\Re(z)^{-2}$. From (14) and part 2 of Lemma 8 it is clear that the absolute difference $\Delta=\left|\mathcal{R}_{z}^{1}-\mathcal{R}_{z}^{2}\right|$ must satisfy

$$
\Delta[G, \circ] \leq \alpha \quad \text { and } \quad \Delta[G, \circ] \leq \beta \sum_{v \sim 0} \Delta[G-\circ, v] .
$$

In turn, each $\Delta[G-\circ, v]$ appearing in the second upper-bound may be further expanded into $\beta \sum_{w \sim v, w \neq \circ} \Delta[G-\circ-v, w]$. Iterating this procedure $k$ times, one obtains $\Delta[G, \circ] \leq \beta^{k} d^{k} \alpha$. Taking the infimum over all $k$ yields $\Delta[G, \circ]=0$, since the assumption $\Re(z)>\sqrt{d}$ means precisely $\beta d<1$.

Proof of Theorem 5. For clarity, we divide the proof in three parts : we first define a specific solution which satisfies (14). We will then prove its unicity and check its continuity. This will prove parts 1-3 of Theorem 5.

Analytic existence. Fix $[G, \circ] \in \mathcal{G}_{d}$, and consider an arbitrary collection of $\mathbb{H}_{+} \rightarrow z \mathbb{H}_{+}$ analytic functions $z \mapsto \mathcal{R}_{z}^{0}[H, i]$, indexed by the elements $[H, i] \in \operatorname{Succ}^{*}[\mathrm{G}, \circ]$. For every $n \geq 1$, define recursively

$$
\mathcal{R}_{z}^{n}[H, i]=z^{2}\left(z^{2}+\sum_{j \sim i} \mathcal{R}_{z}^{n-1}[H-i, j]\right)^{-1}
$$

for all $z \in \mathbb{H}_{+}$and $[H, i] \in \operatorname{Succ}^{*}[\mathrm{G}, \circ]$. By Lemma 8 , each sequence $\left(z \mapsto \mathcal{R}_{z}^{n}[H, i]\right)_{n \in \mathbb{N}}$ is relatively compact in $\mathcal{H}$. Consequently, their joint collection as $[H, i]$ varies in the denumerable set $\operatorname{Succ}^{*}[\mathrm{G}, \circ]$ is relatively compact in the product space $\mathcal{H}^{\mathrm{Succ}^{*}[\mathrm{G}, \mathrm{o}]}$. Passing to the limit in (15), we see that any pre-limit $\mathcal{R}_{z}: \operatorname{Succ}^{*}[\mathrm{G}, \circ] \rightarrow \mathrm{z} \mathbb{H}_{+}$must automatically satisfy (14) for each $z \in \mathbb{H}_{+}$. By Lemma 9, this determines uniquely the value of $\mathcal{R}_{z}[G, \circ]$ for $z$ with sufficiently large real part, and hence everywhere in $\mathbb{H}_{+}$by analyticity. To sum up, we have just proved the following : for every $[G, \circ] \in \mathcal{G}_{d}$, the limit

$$
\mathcal{R}_{z}[G, \circ]:=\lim _{n \rightarrow \infty} \mathcal{R}_{z}^{n}[G, \circ]
$$

exists in $\mathcal{H}$, satisfies the recursion (14), and does not depend upon the choice of the initial condition $\mathcal{R}_{z}^{0}$ : $\operatorname{Succ}^{*}[\mathrm{G}, \mathrm{o}] \rightarrow z \mathbb{H}_{+}$(provided that the latter is analytic in $z \in \mathbb{H}_{+}$).

Pointwise uniqueness. Let us now show that any $\mathcal{S}: \operatorname{Succ}^{*}[\mathrm{G}, \circ] \rightarrow z \mathbb{H}_{+}$satisfying the recursion (14) at a fixed value $z=z_{0} \in \mathbb{H}_{+}$must coincide with the $z=z_{0}$ specialization of the analytic solution constructed above. For each $[H, i] \in \operatorname{Succ}^{*}[\mathrm{G}, \circ]$, the constant initial function $\mathcal{R}_{z}^{0}[H, i]:=\mathcal{S}[H, i]$ is trivially analytic from $\mathbb{H}_{+}$to $z \mathbb{H}_{+}$, so the iteration (15) must converge to the analytic solution $\mathcal{R}_{z}$. Since $\mathcal{R}_{z_{0}}^{n}=\mathcal{S}$ for all $n \in \mathbb{N}$, we obtain $\mathcal{R}_{z_{0}}=\mathcal{S}$, as desired.

Continuity. Finally, assume that $\left(\left[G_{n}, \circ\right]\right)_{n \geq 1} \in \mathcal{G}_{d}^{\mathbb{N}}$ converges locally to $[G, \circ]$, and let us show that

$$
\mathcal{R}_{z}\left[G_{n}, \circ\right] \underset{n \rightarrow \infty}{\stackrel{\mathcal{H}}{\longrightarrow}} \mathcal{R}_{z}[G, \circ]
$$

It is routine that, up to rooted isomorphisms, $G, G_{1}, G_{2}, \ldots$ may be represented on a common vertex set, in such a way that for each fixed $k \in \mathbb{N},\left[G_{n}, \circ\right]_{k}=[G, \circ]_{k}$ for all $n \geq n_{k}$. By construction, any simple path $v_{1} \ldots v_{k}$ starting from the root in $G$ is now also a simple path starting from the root in each $G_{n}, n \geq n_{k}$, so the $\mathcal{H}$-valued sequence $\left(z \mapsto \mathcal{R}_{z}\left[G_{n}-\left\{v_{1}, \ldots, v_{k-1}\right\}, v_{k}\right]\right)_{n \geq n_{k}}$ is well defined, and relatively compact (Lemma 8). Again, the denumerable collection of all sequences obtained by letting the simple path $v_{1} \ldots v_{k}$ vary in $[G, \circ]$ is relatively compact for the product topology, and any pre-limit must by construction satisfy (14). By pointwise uniqueness, the convergence (17) must hold. 


\subsection{Proof of Theorem 6 : convergence of the Boltzmann distribution}

Consider an infinite $[G, \circ] \in \mathcal{G}_{d}$, and let $\left(\left[G_{n}, \circ\right]\right)_{n \geq 1}$ be a sequence of finite rooted connected graphs converging locally to $[G, \circ]$. As above, represent $G, G_{1}, G_{2}, \ldots$ on a common vertex set, in such a way that for each $k \in \mathbb{N},\left[G_{n}, \circ\right]_{k}=[G, \circ]_{k}$ for all $n \geq n_{k}$. Now fix an arbitrary finite matching $M$ in $G$, and denote by $v_{1}, \ldots, v_{2|M|}$ the vertices spanned by $M$, in any order. By construction, $M$ is also a matching of $G_{n}$ for large enough $n$. But the matchings of $G_{n}$ that contain $M$ are in $1-1$ correspondence with the matchings of $G_{n}-\left\{v_{1}, \ldots, v_{2|M|}\right\}$, and hence

$$
\mu_{G_{n}}^{z}(M \subseteq \mathcal{M})=\frac{P_{G_{n}-\left\{v_{1}, \ldots, v_{2|M|}\right\}}(z)}{P_{G_{n}}(z)}=z^{-2 M} \prod_{k=1}^{2 M} \mathcal{R}_{z}\left[G_{n}-\left\{v_{1}, \ldots, v_{k-1}\right\}, v_{k}\right] .
$$

But $\left[G_{n}-\left\{v_{1}, \ldots, v_{k-1}\right\}, v_{k}\right]$ converges locally to $\left[G-\left\{v_{1}, \ldots, v_{k-1}\right\}, v_{k}\right]$, so by continuity of $\mathcal{R}_{z}$,

$$
\mu_{G_{n}}^{z}(M \subseteq \mathcal{M}) \underset{n \rightarrow \infty}{\longrightarrow} z^{-2 M} \prod_{k=1}^{2 M} \mathcal{R}_{z}\left[G-\left\{v_{1}, \ldots, v_{k-1}\right\}, v_{k}\right]
$$

Proof of Corollary 7 . Analytic convergence of the free energy follows from Theorem 6 and Lebesgue dominated convergence Theorem, since for any finite graph $G=(V, E)$ we have

$$
\left(\log P_{G}\right)^{\prime}(z)=\frac{P_{G}^{\prime}(z)}{P_{G}(z)}=\frac{1}{|V|} \sum_{\circ \in V} \frac{\mathcal{R}_{z}[G, \circ]}{z}=\frac{\rho\left[\mathcal{R}_{z}[G, \circ]\right]}{z} .
$$

The uniform domination $\left|\frac{\rho\left[\mathcal{R}_{z}[G, 0]\right]}{z}\right| \leq \frac{1}{\Re(z)}$ is provided by Lemma 8 ,

\section{The zero-temperature limit}

Motivated by the asymptotic study of maximum matchings, we now let the temperature $z \rightarrow 0$.

\subsection{The case of bounded degree}

We first use the results from the previous section to prove a version of Theorem 1 for graphs with bounded degree.

Theorem 10 (The zero temperature limit in graphs with bounded degree) For any $[G, \circ] \in$ $\mathcal{G}_{d}$, the zero temperature limit

$$
\mathcal{R}_{*}[G, \circ]=\lim _{z \rightarrow 0} \downarrow \mathcal{R}_{z}[G, \circ]
$$

exists. Moreover, $\mathcal{R}_{*}: \mathcal{G}_{d} \rightarrow[0,1]$ is the largest solution to the recursion

$$
\mathcal{R}_{*}[G, \circ]=\left(1+\sum_{v \sim \circ}\left(\sum_{w \sim v} \mathcal{R}_{*}[G-\circ-v, w]\right)^{-1}\right)^{-1}
$$

with the conventions $0^{-1}=\infty, \infty^{-1}=0$. When $G$ is finite, $\mathcal{R}_{*}[G, \circ]$ is the probability that $\circ$ is exposed in a uniform maximum matching. 
Proof. Fix $[G, \circ] \in \mathcal{G}_{d}$. First, we claim that $z \mapsto \mathcal{R}_{z}[G, \circ]$ is non-decreasing on $\mathbb{R}_{+}$. Indeed, this is obvious if $G$ is reduced to o, since in that case the REP is simply 1 . It then inductively extends to any finite graph $[G, \circ]$, because iterating twice (14) gives

$$
\mathcal{R}_{z}[G, \circ]=\left(1+\sum_{v \sim \circ}\left(z^{2}+\sum_{w \sim v} \mathcal{R}_{z}[G-\circ-v, w]\right)^{-1}\right)^{-1}
$$

For the infinite case, $[G, \circ]$ is the local limit of the sequence of finite truncations $\left([G, \circ]_{n}\right)_{n \in \mathbb{N}}$, where, for $n \geq 1,[G, \circ]_{n}$ denotes the finite rooted subgraph induced by the vertices lying at graph-distance at most $n$ from $\circ$. So by continuity of the REP, $\mathcal{R}_{z}[G, \circ]=\lim _{n \rightarrow \infty} \mathcal{R}_{z}[G, \circ]_{n}$ must be non-decreasing in $z$ as well. This guarantees the existence of the $[0,1]$-valued limit

$$
\mathcal{R}_{*}[G, \circ]=\lim _{z \rightarrow 0} \downarrow \mathcal{R}_{z}[G, \circ] .
$$

Moreover, taking the $z \rightarrow 0$ limit in (19) guarantees the recursive formula (18).

Finally, consider $\mathcal{S}_{*}: \operatorname{Succ}^{*}[\mathrm{G}, \circ] \rightarrow[0,1]$ satisfying the recursion (18). Let us show by induction over $n \in \mathbb{N}$ that for every $[H, i] \in \operatorname{Succ}^{*}[\mathrm{G}, \circ]$ and $z>0$,

$$
\mathcal{S}_{*}[H, i] \leq \mathcal{R}_{z}[H, i]_{2 n} .
$$

The statement is trivial when $n=0\left(\mathcal{R}_{z}[H, i]_{0}=1\right)$, and is preserved from $n$ to $n+1$ because

$$
\begin{aligned}
\mathcal{R}_{z}[H, i]_{2 n+2} & =\left(1+\sum_{j \sim i}\left(z^{2}+\sum_{k \sim j} \mathcal{R}_{z}[H-i-j, k]_{2 n}\right)^{-1}\right)^{-1} \\
& \geq\left(1+\sum_{j \sim i}\left(\sum_{k \sim j} \mathcal{S}_{*}[H-i-j, k]\right)^{-1}\right)^{-1}=\mathcal{S}_{*}[H-i, j] .
\end{aligned}
$$

Letting $n \rightarrow \infty$ and then $z \rightarrow 0$ in (20) yields $\mathcal{S}_{*} \leq \mathcal{R}_{*}$, which completes the proof

This naturally raises the following question : may the zero temperature limit be interchanged with the infinite volume limit, as suggested by the diagram below?

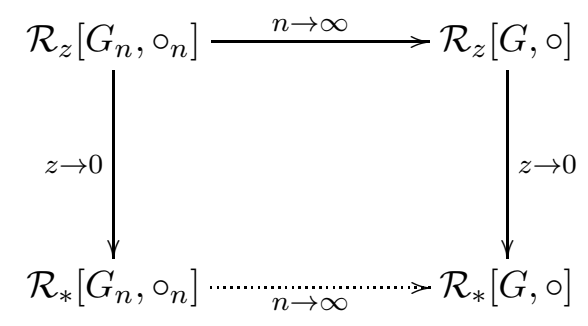

Unfortunately, the recursion (18) may admit several distinct solutions, and this translates as follows : in the limit of zero temperature, correlation decay breaks for the monomer-dimer model, in the precise sense that the functional $\mathcal{R}_{*}: \mathcal{G}_{d} \rightarrow[0,1]$ is no longer continuous with respect to local convergence. For example, one can easily construct an infinite rooted tree $[T, \circ]$ with bounded degree such that

$$
\lim _{n \rightarrow \infty} \downarrow \mathcal{R}_{*}[T, \circ]_{2 n} \neq \lim _{n \rightarrow \infty} \uparrow \mathcal{R}_{*}[T, \circ]_{2 n+1} .
$$


Indeed, consider the case of $T$ being the graph on $\mathbb{N}$ rooted at $0=0$, where two integers share an edge if they differ by 1 . Then, a straightforward computation gives $\mathcal{R}_{*}[T, \circ]_{2 n}=1 / 2$ while $\mathcal{R}_{*}[T, \circ]_{2 n+1}=0$. Despite this lack of correlation decay, the interchange of limits turns out to be valid "on average", i.e. when looking at a uniformly chosen vertex $\circ$.

Theorem 11 (The limiting matching number of bounded-degree graph sequences) Let $\rho$ be a probability distribution over $\mathcal{G}_{d}$. For any sequence of finite graphs $\left(G_{n}=\left(V_{n}, E_{n}\right)\right)_{n \in \mathbb{N}}$ satisfying $\left|E_{n}\right|=O\left(\left|V_{n}\right|\right)$ and having $\rho$ as a random weak limit,

$$
\frac{\nu\left(G_{n}\right)}{\left|V_{n}\right|} \underset{n \rightarrow \infty}{\longrightarrow} \frac{1-\mathbb{E}_{\rho}\left[\mathcal{R}_{*}\right]}{2} .
$$

In order to get our Theorem 1, we need to remove the bounded degree assumption. This is done below. In the case where the limit $\rho$ is a (two-level) Galton-Watson tree, the recursion (18) simplifies into a recursive distributional equation (RDE). The computations for these cases are done in Section 5 ,

Proof of Theorem 11.

Let $G=(V, E)$ be a finite graph and $M$ be any maximal matching of $G$. Then

$$
\sum_{v \in V} \mathbf{1}(v \text { is exposed in } M)=|V|-2 \sum_{e \in E} \mathbf{1}(e \in M) .
$$

In particular, if $\rho=\mathcal{U}(G)$, we have the elementary identity

$$
\mathbb{E}_{\rho}\left[\mathcal{R}_{*}\right]=1-\frac{2 \nu(G)}{|V|}
$$

The proof of Theorem 11 will easily follow from the following uniform control:

Lemma 12 (Uniform continuity around the zero-temperature point) Let $G=(V, E)$ be a finite graph. For any $0<z<1$,

$$
\mathbb{E}_{\rho}\left[\mathcal{R}_{z}\right]+\frac{|E|}{|V|} \frac{\log 2}{\log z} \leq \mathbb{E}_{\rho}\left[\mathcal{R}_{*}\right] \leq \mathbb{E}_{\rho}\left[\mathcal{R}_{z}\right]
$$

Indeed, let $\rho$ be a probability distribution on $\mathcal{G}_{d}$, and let $\left(G_{n}=\left(V_{n}, E_{n}\right)\right)_{n \in \mathbb{N}}$ be a sequence of finite graphs with $\left|V_{n}\right|=O\left(\left|E_{n}\right|\right)$, whose random weak limit is $\rho$. For each $n \in \mathbb{N}$, set $\rho_{n}=\mathcal{U}\left(G_{n}\right)$. With these notations, proving Theorem 11 amounts to establish :

$$
\mathbb{E}_{\rho_{n}}\left[\mathcal{R}_{*}\right] \underset{n \rightarrow \infty}{\longrightarrow} \mathbb{E}_{\rho}\left[\mathcal{R}_{*}\right]
$$

However, since $\rho_{n} \Longrightarrow \rho$, and since each $\mathcal{R}_{z}, z>0$ is continuous and bounded, we have for every $z>0$

$$
\mathbb{E}_{\rho_{n}}\left[\mathcal{R}_{z}\right] \underset{n \rightarrow \infty}{\longrightarrow} \mathbb{E}_{\rho}\left[\mathcal{R}_{z}\right]
$$

Thus, setting $C=\sup _{n \in \mathbb{N}} \frac{\left|E_{n}\right|}{\left|V_{n}\right|}$ and letting $n \rightarrow \infty$ in (22), we see that for any $z<1$,

$$
\mathbb{E}_{\rho}\left[\mathcal{R}_{z}\right]+C \frac{\log 2}{\log z} \leq \liminf _{n \rightarrow \infty} \mathbb{E}_{\rho_{n}}\left[\mathcal{R}_{*}\right] \leq \limsup _{n \rightarrow \infty} \mathbb{E}_{\rho_{n}}\left[\mathcal{R}_{*}\right] \leq \mathbb{E}_{\rho}\left[\mathcal{R}_{z}\right]
$$


Letting finally $z \rightarrow 0$, we obtain exactly (23), and it only remains to show Lemma 12 ,

Proof of Lemma [12. Fix $0<z<1$. Since $z \mapsto \mathbb{E}_{\rho}\left[\mathcal{R}_{z}\right]$ is non-decreasing, we have

$$
\mathbb{E}_{\rho}\left[\mathcal{R}_{*}\right] \leq \mathbb{E}_{\rho}\left[\mathcal{R}_{z}\right] \leq \frac{-1}{\log z} \int_{z}^{1} s^{-1} \mathbb{E}_{\rho}\left[\mathcal{R}_{s}\right] d s
$$

Use $\mathbb{E}_{\rho}\left[\mathcal{R}_{s}\right]=\frac{s P_{G}^{\prime}(s)}{|V| P_{G}(s)}$ to rewrite this as

$$
\mathbb{E}_{\rho}\left[\mathcal{R}_{*}\right] \leq \mathbb{E}_{\rho}\left[\mathcal{R}_{z}\right] \leq \frac{1}{|V| \log z} \log \frac{P_{G}(z)}{P_{G}(1)}
$$

Now, $P_{G}(1)$ is the total number of matchings and is thus clearly at most $2^{|E|}$, while $P_{G}(z)$ is at least $z^{|V|-2 \nu(G)}$. Using (21), these two bounds yield to

$$
\mathbb{E}_{\rho}\left[\mathcal{R}_{*}\right] \leq \mathbb{E}_{\rho}\left[\mathcal{R}_{z}\right] \leq \frac{1}{|V| \log z}\left(|V| \mathbb{E}_{\rho}\left[\mathcal{R}_{*}\right] \log z-|E| \log 2\right)
$$

This gives (22).

\subsection{The case of unbounded degree}

In this section, we establish Theorem 1 in full generality, removing the restriction of bounded degree from Theorem [11. To this end, we introduce the $d$-truncation $G^{d}(d \in \mathbb{N})$ of a graph $G=(V, E)$, obtained from $G$ by isolating all vertices with degree more than $d$, i.e. removing any edge incident to them. This transformation is clearly continuous with respect to local convergence. Moreover, its effect on the matching number can be easily controlled :

$$
\nu\left(G^{d}\right) \leq \nu(G) \leq \nu\left(G^{d}\right)+\#\left\{v \in V ; \operatorname{deg}_{G}(v)>d\right\} .
$$

Now, consider a sequence of finite graphs $\left(G_{n}\right)_{n \in \mathbb{N}}$ admitting a random weak limit $(G, \circ)$. First, fixing $d \in \mathbb{N}$, we may apply Theorem 11 to the sequence $\left(G_{n}^{d}\right)_{n \in \mathbb{N}}$ to obtain :

$$
\frac{\nu\left(G_{n}^{d}\right)}{\left|V_{n}\right|} \underset{n \rightarrow \infty}{\longrightarrow} \frac{1-\mathbb{E}_{\rho_{d}}\left[\mathcal{R}_{*}\right]}{2},
$$

where $\rho_{d}$ is the $d$-truncation of $\rho$. Second, we may rewrite (24) as

$$
\left|\frac{\nu\left(G_{n}^{d}\right)}{\left|V_{n}\right|}-\frac{\nu\left(G_{n}\right)}{\left|V_{n}\right|}\right| \leq \frac{\#\left\{v \in V_{n} ; \operatorname{deg}_{G_{n}}(v)>d\right\}}{\left|V_{n}\right|} .
$$

Letting $n \rightarrow \infty$, we obtain

$$
\limsup _{n \rightarrow \infty}\left|\frac{1-\rho_{d}\left[\mathcal{R}_{*}\right]}{2}-\frac{\nu\left(G_{n}\right)}{\left|V_{n}\right|}\right| \leq \rho(\operatorname{deg}(\circ)>d),
$$

This last line is, by an elementary application of Cauchy criterion, enough to guarantee the convergence promised by Theorem 1, i.e.

$$
\frac{\nu\left(G_{n}\right)}{\left|V_{n}\right|} \underset{n \rightarrow \infty}{\longrightarrow} \gamma, \quad \text { where } \quad \gamma:=\lim _{d \rightarrow \infty} \frac{1-\mathbb{E}_{\rho_{d}}\left[\mathcal{R}_{*}\right]}{2} .
$$


Note that because of the possible absence of correlation decay, the largest solution $\mathcal{R}_{*}[G, \circ]$ is not a continuous function of $(G, \circ) \in \mathcal{G}$. In particular, we do not know whether it is always the case that

$$
\gamma=\frac{1-\mathbb{E}_{\rho}\left[\mathcal{R}_{*}\right]}{2}
$$

as established in Theorem 11 for graphs with bounded degree. However, (26) holds in the particular cases where we have an explicit formula for $\mathbb{E}_{\rho}\left[\mathcal{R}_{*}\right]$ which depends continuously upon the degree distribution as will be the case in Section 5 .

\section{Computations on (hierarchal) Galton-Watson trees}

\subsection{The case of Galton-Watson trees}

We now investigate the special case where the limiting random graph is a UGW tree $T$. Specifically, we fix a distribution $\pi \in \mathcal{P}(\mathbb{N})$ with finite support (we will relax this assumption in the sequel) and we consider a UGW tree $T$ with degree distribution $\pi$ as defined in Section 2 , The random matchings $\mathcal{M}_{T}^{z}, z \geq 0$ are perfectly well-defined, and all the previously established results for graphs with bounded degree hold almost surely. However, the self-similar recursive structure of $T$ gives to the fixed-point characterizations (14) and (18) a very special form that is worth making explicit.

Before we start, let us insist on the fact that $\mathcal{R}_{z}[T](z>0)$ is random : it is the quenched probability that the root is exposed at temperature $z$, given the random tree $T$. In light of Theorem 1, it becomes important to ask for its distribution. Let $\mathcal{P}([0,1])$ denote the space of Borel probability measures on $[0,1]$. Given $z>0, \nu \in \mathcal{P}(\mathbb{N})$ and $\mu \in \mathcal{P}([0,1])$, we denote by $\Theta_{\nu, z}(\mu)$ the law of the $[0,1]-$ valued r.v.

$$
Y=\frac{z^{2}}{z^{2}+\sum_{i=1}^{\mathcal{N}} X_{i}}
$$

where $\mathcal{N} \sim \nu$ and $X_{1}, X_{2}, \ldots \sim \mu$, all of them being independent. This defines an operator $\Theta_{\nu, z}$ on $\mathcal{P}([0,1])$. The corresponding fixed point equation $\mu=\Theta_{\nu, z}(\mu)$ belongs to the general class of recursive distributional equations, or RDE. Equivalently, it can be rewritten as

$$
X \stackrel{d}{=} \frac{z^{2}}{z^{2}+\sum_{i=1}^{\mathcal{N}} X_{i}},
$$

where $X_{1}, X_{2}, \ldots$ are i.i.d. copies of the unknown random variable $X$. Note that the same RDE appears in the analysis of the spectrum and rank of adjacency matrices of random graphs [11], [12. With this notations in hands, the infinite system of equations (14) defining $\mathcal{R}_{z}[T]$ clearly leads to the following distributional characterization:

Lemma 13 For any $z>0, \mathcal{R}_{z}[T]$ has distribution $\Theta_{\pi, z}\left(\mu_{z}\right)$, where $\mu_{z}$ is solution to the $R D E$ $\mu_{z}=\Theta_{\widehat{\pi}, z}\left(\mu_{z}\right)$.

The same program can be carried out in the zero temperature limit. Specifically, given $\nu, \nu^{\prime} \in \mathcal{P}(\mathbb{N})$ and $\mu \in \mathcal{P}([0,1])$, we define $\Theta_{\nu, \nu^{\prime}}(\mu)$ as the law of the $[0,1]$-valued r.v.

$$
Y=\frac{1}{1+\sum_{i=1}^{\mathcal{N}}\left(\sum_{j=1}^{\mathcal{N}_{i}{ }^{\prime}} X_{i j}\right)^{-1}},
$$


where $\mathcal{N} \sim \nu, \mathcal{N}_{i}^{\prime} \sim \nu^{\prime}$, and $X_{i j} \sim \mu$, all of them being independent. This defines an operator $\Theta_{\nu, \nu^{\prime}}$ on $\mathcal{P}([0,1])$ whose fixed points will play a crucial role in our study. Then, Theorem 10 implies:

Lemma 14 The random variable $\mathcal{R}_{*}[T]$ has law $\Theta_{\pi, \widehat{\pi}}\left(\mu_{*}\right)$, where $\mu_{*}$ is the largest solution to the $R D E \mu_{*}=\Theta_{\widehat{\pi}, \widehat{\pi}}\left(\mu_{*}\right)$.

Recall that the mean of $\Theta_{\pi, \widehat{\pi}}\left(\mu_{*}\right)$ gives precisely the asymptotic size of a maximum matching for any sequence of finite random graphs whose random weak limit is $T$ (Theorem 11). We will solve this RDE in the next section in the more general set-up of UHGW trees. Combined with Theorem 1 and a simple continuity argument to remove the bounded degree assumption, this will prove Theorem 2 ,

\subsection{The case of hierarchal Galton-Watson trees}

As in previous section, we first assume that both $\pi^{a}$ and $\pi^{b}$ have a finite support. We can define a RDE but with some care about the types $a$ and $b$. The corresponding results read as follows:

Lemma 15 For any $z>0$, conditionally on the root being of type $b$ (resp. a), $\mathcal{R}_{z}[T]$ has distribution $\Theta_{\pi^{b}, z}\left(\mu_{z}^{a}\right)$ (resp. $\Theta_{\pi^{b}, z}\left(\mu_{z}^{b}\right)$ ), where $\mu_{z}^{a}$ is solution to the RDE:

$$
\mu_{z}^{a}=\Theta_{\widehat{\pi}^{a}, z} \circ \Theta_{\widehat{\pi}^{b}, z}\left(\mu_{z}^{a}\right)
$$

and $\mu_{z}^{b}=\Theta_{\widehat{\pi}^{b}, z}\left(\mu_{z}^{a}\right)$.

For $z=0$ : conditionally on the root being of type a (resp. b), the random variable $\mathcal{R}_{*}[T]$ has law $\Theta_{\pi^{a}, \widehat{\pi}^{b}}\left(\mu_{*}^{a}\right)$ (resp. $\left.\Theta_{\pi^{b}, \widehat{\pi}^{a}}\left(\mu_{*}^{b}\right)\right)$, where $\mu_{*}^{a}$ is the largest solution to the RDE

$$
\mu_{*}^{a}=\Theta_{\widehat{\pi}^{a}, \widehat{\pi}^{b}}\left(\mu_{*}^{a}\right)
$$

and $\mu_{*}^{b}$ is the largest solution to the $R D E \mu_{*}^{b}=\Theta_{\widehat{\pi}^{b}, \widehat{\pi}^{a}}\left(\mu_{*}^{b}\right)$.

We now analyze the RDE (28). We define:

$$
F^{a}(x)=\phi^{a}\left(1-\widehat{\phi}^{b}(1-x)\right)-\frac{\phi^{a \prime}(1)}{\phi^{b \prime}(1)}\left(1-\phi^{b}(1-x)-x \phi^{b \prime}(1-x) .\right)
$$

Observe that

$$
F^{a \prime}(x)=\frac{\phi^{a \prime}(1)}{\phi^{b \prime}(1)} \phi^{b \prime \prime}(1-x)\left(\widehat{\phi}^{a}\left(1-\widehat{\phi}^{b}(1-x)\right)-x\right)
$$

Hence any $x$ where $F^{a}$ admits a local maximum must satisfy $x=\widehat{\phi}^{a}\left(1-\widehat{\phi}^{b}(1-x)\right)$. We define the historical records of $F^{a}$ as the set of $x \in[0,1]$ such that $x=\widehat{\phi}^{a}\left(1-\widehat{\phi}^{b}(1-x)\right)$ and for any $0 \leq y<x, F^{a}(x)>F^{a}(y)$ (the latter condition being empty if $x=0$ ).

Theorem 16 If $p_{1}<\ldots<p_{r}$ are the locations of the historical records of $F^{a}$, then the $R D E$ (28) admits exactly $r$ solutions ; moreover, these solutions can be stochastically ordered, say $\mu_{1}<\ldots<\mu_{r}$, and for any $i \in\{1, \ldots, r\}$, 
- $\mu_{i}((0,1])=p_{i}$;

- $\Theta_{\pi^{a}, \hat{\pi}^{b}}\left(\mu_{i}\right)$ has mean $F^{a}\left(p_{i}\right)$.

The proof of Theorem 16 relies on two lemmas.

Lemma 17 The operators $\Theta_{\pi^{a}, \widehat{\pi}^{b}}$ and $\Theta_{\widehat{\pi}^{a}, \widehat{\pi}^{b}}$ are continuous (with respect to weak convergence) and strictly increasing (with respect to stochastic ordering) on $\mathcal{P}([0,1])$.

Proof of Lemma 17. It follows directly from the fact that, for any $n \geq 0$ and any $n_{1}, \ldots, n_{n} \geq$ 0 , the mapping

$$
x \mapsto \frac{1}{1+\sum_{i=1}^{n}\left(\sum_{j=1}^{n_{i}} x_{i j}\right)^{-1}}
$$

is continuous and increasing from $[0,1]^{n_{1}+\ldots+n_{n}}$ to $[0,1]$.

Lemma 18 For any $\mu \in \mathcal{P}([0,1])$, letting $p=\mu((0,1])$, we have

1. $\Theta_{\widehat{\pi}^{a}, \widehat{\pi}^{b}}(\mu)((0,1])=\widehat{\phi}^{a}\left(1-\widehat{\phi}^{b}(1-p)\right)$

2. if $\Theta_{\widehat{\pi}^{a}, \widehat{\pi}^{b}}(\mu) \leq \mu$, then the mean of $\Theta_{\pi^{a}, \widehat{\pi}^{b}}(\mu)$ is at least $F^{a}(p)$.

3. if $\Theta_{\widehat{\pi}^{a}, \widehat{\pi}^{b}}(\mu) \geq \mu$, then the mean of $\Theta_{\pi^{a}, \widehat{\pi}^{b}}(\mu)$ is at most $F^{a}(p)$;

In particular, if $\mu$ is a fixed point of $\Theta_{\widehat{\pi}^{a}, \widehat{\pi}^{b}}$, then $p=\widehat{\phi}^{a}\left(1-\widehat{\phi}^{b}(1-p)\right)$ and $\Theta_{\pi^{a}, \widehat{\pi}^{b}}(\mu)$ has mean $F^{a}(p)$.

Proof of Lemma 18. In equation (27) it is clear that $Y>0$ if and only if for any $i \in\{1, \ldots, \mathcal{N}\}$, there exists $j \in\left\{1, \ldots, \mathcal{N}_{i}^{\prime}\right\}$ such that $X_{i j}>0$. With the notation introduced above, this rewrites:

$$
\Theta_{\widehat{\pi}^{a}, \widehat{\pi}^{b}}(\mu)((0,1])=\widehat{\phi}^{a}\left(1-\widehat{\phi}^{b}(1-\mu((0,1]))\right),
$$

hence the first result follows.

Now let $X \sim \mu, Y \sim \Theta_{\widehat{\pi}^{a}, \widehat{\pi}^{b}}(\mu), \mathcal{N}^{a} \sim \pi^{a}, \widehat{\mathcal{N}}^{a} \sim \widehat{\pi}^{a}$, and let $S, S_{1}, \ldots$ have the distribution of the sum of a $\widehat{\pi}^{b}$-distributed number of i.i.d. copies of $X$, all these variables being independent. Observe that

$$
\begin{aligned}
\frac{1}{1+\sum_{i=1}^{\mathcal{N}^{a}} S_{i}^{-1}} & =\left(1-\frac{\sum_{i=1}^{\mathcal{N}^{a}} S_{i}^{-1}}{1+\sum_{i=1}^{\mathcal{N}^{a}} S_{i}^{-1}}\right) \mathbf{1}_{\left\{\forall i=1 \ldots \mathcal{N}^{a}, S_{i}>0\right\}} \\
& =\mathbf{1}_{\left\{\forall i=1 \ldots \mathcal{N}^{a}, S_{i}>0\right\}}-\sum_{j=1}^{\mathcal{N}^{a}} \frac{S_{j}^{-1}}{1+S_{j}^{-1}+\sum_{1 \leq i \leq \mathcal{N}^{a}, i \neq j} S_{i}^{-1}} \mathbf{1}_{\left\{\forall i=1 \ldots \mathcal{N}^{a}, S_{i}>0\right\}}
\end{aligned}
$$

Then, $\Theta_{\pi^{a}, \widehat{\pi}^{b}}(\mu)$ has mean

$$
\begin{aligned}
\mathbb{E}\left[\frac{1}{1+\sum_{i=1}^{\mathcal{N}^{a}} S_{i}^{-1}}\right] & =\mathbb{P}\left(\forall i=1 \ldots \mathcal{N}^{a}, S_{i}>0\right)-\sum_{k=1}^{\infty} k \pi_{k}^{a} \mathbb{E}\left[\frac{S^{-1}}{S^{-1}+1+\sum_{i=1}^{k-1} S_{i}^{-1}} \mathbf{1}_{\left\{S>0, \forall i=1 \ldots k-1, S_{i}>0\right\}}\right] \\
& =\phi^{a}\left(1-\widehat{\phi}^{b}(1-p)\right)-\phi^{a \prime}(1) \mathbb{E}\left[\frac{S^{-1}}{S^{-1}+1+\sum_{i=1}^{\hat{\mathcal{N}}^{a}} S_{i}^{-1}} \mathbf{1}_{\left\{S>0, \forall i=1 \ldots \hat{\mathcal{N}}^{a}, S_{i}>0\right\}}\right] \\
& =\phi^{a}\left(1-\widehat{\phi}^{b}(1-p)\right)-\phi^{a \prime}(1) \mathbb{E}\left[\frac{Y}{Y+S} \mathbf{1}_{\{S>0\}}\right]
\end{aligned}
$$


where the second and last lines follow from (515) and $Y \sim \Theta_{\widehat{\pi}^{a}, \widehat{\pi}^{b}}(\mu)$, respectively. Now, for any $s>0, x \mapsto x / x+s$ is increasing and hence, depending on whether $\Theta_{\widehat{\pi}, \widehat{\pi}}(\mu) \geq \mu$ or $\Theta_{\widehat{\pi}^{a}, \widehat{\pi}^{b}}(\mu) \leq \mu$, $\Theta_{\pi^{a}, \widehat{\pi}^{b}}(\mu)$ has mean at most/least:

$\phi^{a}\left(1-\widehat{\phi}^{b}(1-p)\right)-\phi^{a \prime}(1) \mathbb{E}\left[\frac{X}{X+S} \mathbf{1}_{\{S>0\}}\right]=\phi^{a}\left(1-\widehat{\phi}^{b}(1-p)\right)-\phi^{a \prime}(1) \mathbb{E}\left[\frac{X}{X+\sum_{i=1}^{\hat{\mathcal{N}}^{b}} X_{i}} \mathbf{1}_{\left\{\mathcal{N}^{*} \geq 1\right\}}\right]$,

with $X_{i}$ are i.i.d. copies of $X$ independent of $\widehat{\mathcal{N}}^{b} \sim \widehat{\pi}^{b}$ and $\mathcal{N}^{*}=\sum_{i=1}^{\widehat{\mathcal{N}}^{b}} \mathbf{1}_{\left\{X_{i}>0\right\}}$. Now if $X^{\prime}$ is the law of $X$ conditioned on $\{X>0\}$, and $X_{i}^{\prime}$ are i.i.d. copies of $X^{\prime}$, by exchangeability, we find

$$
\mathbb{E}\left[\frac{X}{X+\sum_{i=1}^{\hat{\mathcal{N}}^{b}} X_{i}} \mathbf{1}_{\left\{\mathcal{N}^{*} \geq 1\right\}}\right]=p \mathbb{E}\left[\frac{X^{\prime}}{X^{\prime}+\sum_{i=1}^{\mathcal{N}^{*}} X_{i}^{\prime}} \mathbf{1}_{\left\{\mathcal{N}^{*} \geq 1\right\}}\right]=p \mathbb{E}\left[\frac{1}{1+\mathcal{N}^{*}} \mathbf{1}_{\left\{\mathcal{N}^{*} \geq 1\right\}}\right] .
$$

Hence finally, depending on whether $\Theta_{\widehat{\pi}, \widehat{\pi}}(\mu) \geq \mu$ or $\Theta_{\widehat{\pi}^{a}, \widehat{\pi}^{b}}(\mu) \leq \mu, \Theta_{\pi^{a}, \widehat{\pi}^{b}}(\mu), \Theta_{\pi^{a}, \widehat{\pi}^{b}}(\mu)$ has mean at most/least:

$$
\phi^{a}\left(1-\widehat{\phi}^{b}(1-p)\right)-p \phi^{a \prime}(1) \mathbb{E}\left[\frac{1}{1+\mathcal{N}^{*}} \mathbf{1}_{\left\{\mathcal{N}^{*} \geq 1\right\}}\right]
$$

But using the definition (5) and the combinatorial identity $(n+1)\left(\begin{array}{l}n \\ d\end{array}\right)=(d+1)\left(\begin{array}{l}n+1 \\ d+1\end{array}\right)$, one easily derive :

$$
\begin{aligned}
& \phi^{a}\left(1-\widehat{\phi}^{b}(1-p)\right)-p \phi^{a \prime}(1) \mathbb{E}\left[\frac{1}{1+\mathcal{N}^{*}} \mathbf{1}_{\left\{\mathcal{N}^{*} \geq 1\right\}}\right] \\
= & \phi^{a}\left(1-\widehat{\phi}^{b}(1-p)\right)-p \phi^{a \prime}(1) \sum_{n \geq 1}{\widehat{\pi_{n}}}^{b} \sum_{d=1}^{n}\left(\begin{array}{l}
n \\
d
\end{array}\right) \frac{p^{d}(1-p)^{n-d}}{d+1}=F^{a}(p) .
\end{aligned}
$$

Proof of Theorem 16. Let $p \in[0,1]$ such that $\widehat{\phi}^{a}\left(1-\widehat{\phi}^{b}(1-p)\right)=p$, and define $\mu_{0}=$ $\operatorname{Bernoulli}(p)$. From Lemma 18 we know that $\Theta_{\widehat{\pi}^{a}, \widehat{\pi}^{b}}\left(\mu_{0}\right)((0,1])=p$, and since Bernoulli(p) is the largest element of $\mathcal{P}([0,1])$ putting mass $p$ on $(0,1]$, we have $\Theta_{\widehat{\pi}^{a}, \widehat{\pi}^{b}}\left(\mu_{0}\right) \leq \mu_{0}$. Immediately, Lemma 17 guarantees that the limit

$$
\mu_{\infty}=\lim _{k \rightarrow \infty} \searrow \Theta_{\widehat{\pi}^{a}, \widehat{\pi}^{b}}^{k}\left(\mu_{0}\right)
$$

exists in $\mathcal{P}([0,1])$ and is a fixed point of $\Theta_{\widehat{\pi}^{a}, \widehat{\pi}^{b}}$. Moreover, by Fatou's lemma, the number $p_{\infty}=\mu_{\infty}((0,1])$ must satisfy $p_{\infty} \leq p$. But then the mean of $\Theta_{\pi^{a}, \widehat{\pi}^{b}}\left(\mu_{\infty}\right)$ must be both

- equal to $F^{a}\left(p_{\infty}\right)$ by Lemma 18 with $\mu=\mu_{\infty}$;

- at least $F^{a}(p)$ since this holds for all $\Theta_{\pi^{a}, \widehat{\pi}^{b}} \circ \Theta_{\widehat{\pi}^{a}, \widehat{\pi}^{b}}^{k}\left(\mu_{0}\right), k \geq 1$ (Lemma 18 with $\mu=$ $\left.\Theta_{\widehat{\pi}^{a}, \widehat{\pi}^{b}}^{k}\left(\mu_{0}\right)\right)$.

We have just shown both $F^{a}(p) \leq F^{a}\left(p_{\infty}\right)$ and $p_{\infty} \leq p$. From this, we now deduce the one-toone correspondence between historical records of $F^{a}$ and fixed points of $\Theta_{\widehat{\pi}^{a}, \widehat{\pi}^{b}}$. We treat each inclusion separately: 
1. If $F^{a}$ admits an historical record at $p$, then clearly $p_{\infty}=p$, so $\mu_{\infty}$ is a fixed point satisfying $\mu_{\infty}((0,1])=p$.

2. Conversely, considering a fixed point $\mu$ with $\mu((0,1])=p$, we want to deduce that $F^{a}$ admits an historical record at $p$. We first claim that $\mu$ is the above defined limit $\mu_{\infty}$. Indeed, $\mu \leq$ Bernoulli(p) implies $\mu \leq \mu_{\infty}\left(\Theta_{\widehat{\pi}^{a}, \widehat{\pi}^{b}}\right.$ is increasing $)$, and in particular $p \leq p_{\infty}$. Therefore, $p=p_{\infty}$ and $F^{a}(p)=F^{a}\left(p_{\infty}\right)$. In other words, the two ordered distributions $\Theta_{\pi^{a}, \widehat{\pi}^{b}}(\mu) \leq \Theta_{\pi^{a}, \widehat{\pi}^{b}}\left(\mu_{\infty}\right)$ share the same mean, hence are equal. This ensures $\mu=\mu_{\infty}$. Now, if $q<p$ is any historical record location, we know from part 1 that

$$
\nu_{\infty}=\lim _{k \rightarrow \infty} \searrow \Theta_{\widehat{\pi}^{a}, \widehat{\pi}^{b}}^{k}(\operatorname{Bernoulli}(\mathrm{q}))
$$

is a fixed point of $\Theta_{\widehat{\pi}^{a}, \widehat{\pi}^{b}}$ satisfying $\nu_{\infty}((0,1])=q$. But $q<p$, so $\operatorname{Bernoulli}(\mathrm{q})<$ $\operatorname{Bernoulli}(\mathrm{p})$, hence $\nu_{\infty} \leq \mu_{\infty}$. Moreover, this limit inequality is strict because $\nu_{\infty}((0,1])=$ $q<p=\mu_{\infty}((0,1])$. Consequently, $\Theta_{\pi^{a}, \widehat{\pi}^{b}}\left(\nu_{\infty}\right)<\Theta_{\pi^{a}, \widehat{\pi}^{b}}\left(\mu_{\infty}\right)$ and taking expectations, $F^{a}(q)<F^{a}(p)$. Thus, $F^{a}$ admits an historical record at $p$.

We may now finish the proof of Theorem 3 ,

Proof of Theorem 3 : case of bounded degrees. We assume that $\pi_{a}$ and $\pi_{b}$ have bounded support. Recall (7), so that we have $\lambda=\frac{\phi^{b \prime}(1)}{\phi^{a \prime}(1)+\phi^{b \prime}(1)}$, where $\lambda$ is the probability that the root is of type $a$. Theorems 11 and 16 and Lemma 15 give:

$$
\frac{\nu\left(G_{n}\right)}{\left|V_{n}\right|} \underset{n \rightarrow \infty}{\longrightarrow} \frac{\lambda\left(1-\max _{x \in[0,1]} F^{a}(x)\right)+(1-\lambda)\left(1-\max _{x \in[0,1]} F^{b}(x)\right)}{2}
$$

where $F^{a}$ is defined in (29) and $F^{b}$ is defined similarly by

$$
F^{b}(x)=\phi^{b}\left(1-\widehat{\phi}^{a}(1-x)\right)-\frac{\phi^{b \prime}(1)}{\phi^{a \prime}(1)}\left(1-\phi^{a}(1-x)-x \phi^{a \prime}(1-x)\right) .
$$

For any $x$ which is an historical record of $F^{a}$, we define $y=\widehat{\phi}^{b}(1-x)$ so that $\widehat{\phi}^{a}(1-y)=x$. Then we have:

$$
\begin{aligned}
\lambda\left(1-F^{a}(x)\right) & =\lambda\left(1-\phi^{a}(1-y)+\phi^{a \prime}(1)\left(\frac{1}{\phi^{b \prime}(1)}-\frac{\phi^{b}\left(1-\widehat{\phi}^{a}(1-y)\right)}{\phi^{b \prime}(1)}-y \widehat{\phi}^{a}(1-y)\right)\right) \\
& =(1-\lambda)\left(1-F^{b}(y)\right) .
\end{aligned}
$$

By symmetry, this directly implies that $\lambda\left(1-\max _{x \in[0,1]} F^{a}(x)\right)=(1-\lambda)\left(1-\max _{x \in[0,1]} F^{b}(x)\right)$ so that (30) is equivalent to (8). This proves Theorems 2 and 3 for distributions with bounded support.

Proof of Theorem 3 : general case. To keep notation simple, we only prove Theorem 2 . The following proof clearly extends to the case of UHGW trees. Let $G_{1}, G_{2}, \ldots$ be finite random graphs whose local weak limit is a Galton-Watson tree $T$, and assume that the degree distribution $\pi$ of $T$ (with generating function $\phi$ ) has a finite mean : $\phi^{\prime}(1)=\sum_{n} n \pi_{n}<\infty$. For any rooted graph $G$ and any fixed integer $d \geq 1$, recall that $G^{d}$ is the graph obtained from $G$ by deleting 
all edges adjacent to a vertex $v$ whenever $\operatorname{deg}(v)>d$. Hence $T^{d}$ is a Galton-Watson tree whose degree distribution $\pi^{d}$ is defined by

$$
\forall i \geq 0, \pi_{i}^{d}=\pi_{i} \mathbf{1}_{i \leq d}+\mathbf{1}_{i=0} \sum_{k \geq d+1} \pi_{k}
$$

By Theorem 1, Equation (25) and our weaker version of Theorem 2 for distributions with bounded support,

$$
\frac{\nu\left(G_{n}\right)}{\left|V_{n}\right|} \underset{n \rightarrow \infty}{\longrightarrow} \lim _{d \rightarrow \infty} \min _{x \in[0,1]} g^{d}(x)
$$

with $\phi_{d}(x)=\sum_{k=0}^{d} \pi_{k} x^{k}$ and

$$
g^{d}(x)=1-\frac{1}{2}(1-x) \phi_{d}^{\prime}(x)-\frac{1}{2} \phi_{d}(x)-\frac{1}{2} \phi_{d}\left(1-\frac{\phi_{d}^{\prime}(x)}{\phi_{d}^{\prime}(1)}\right) .
$$

Also, as $d \rightarrow \infty$, we have $\phi_{d} \rightarrow \phi$ and $\phi_{d}^{\prime} \rightarrow \phi^{\prime}$ uniformly on $[0,1]$, so

$$
\min _{x \in[0,1]} g^{d}(x) \underset{n \rightarrow \infty}{\longrightarrow} \min _{x \in[0,1]} g(x),
$$

with $g(x)=1-\frac{1}{2}(1-x) \phi^{\prime}(x)-\frac{1}{2} \phi(x)-\frac{1}{2} \phi\left(1-\frac{\phi^{\prime}(x)}{\phi^{\prime}(1)}\right)$. Finally, combining (32) and (33), we easily obtain the desired

$$
\frac{\nu\left(G_{n}\right)}{\left|V_{n}\right|} \underset{n \rightarrow \infty}{\longrightarrow} \min _{x \in[0,1]} g(x) .
$$

\subsection{Proof of Corollary 4}

Note that in Corollary 4, we divide $\nu\left(G_{n}\right)$ by $\left|V_{n}^{a}\right|=\lfloor\alpha m\rfloor$ instead of $\left|V_{n}^{a}\right|+\left|V_{n}^{b}\right|=\lfloor\alpha m\rfloor+m$, so that by Theorem 3, we have $\frac{\nu\left(G_{n}\right)}{\left|V_{n}^{a}\right|} \underset{n \rightarrow \infty}{\longrightarrow} \min _{t \in[0,1]} 1-F^{a}(t)$. We have $\phi^{a}(x)=x^{k}, \phi^{b}(x)=$ $e^{\alpha k(x-1)}$ so that we have:

$$
\begin{aligned}
& F^{a}(x)=\left(1-e^{-k \alpha x}\right)^{k}-\frac{1}{\alpha}\left(1-e^{-k \alpha x}-k \alpha x e^{-k \alpha x}\right) \\
& F^{a \prime}(x)=k^{2} \alpha e^{-k \alpha x}\left(\left(1-e^{-k \alpha x}\right)^{k-1}-x\right) .
\end{aligned}
$$

Let $x^{*}$ be defined as in Corollary 4 as the largest solution to $x=\left(1-e^{-k \alpha x}\right)^{k-1}$. It is easy to check (see Section 6 in [23] for a more general analysis) that

$$
\min _{t \in[0,1]} 1-F^{a}(t)=\min \left\{1,1-F^{a}\left(x^{*}\right)\right\}
$$

Setting $\xi^{*}=k \alpha x^{*}$, we have $\frac{\xi^{*}}{k \alpha}=\left(1-e^{-\xi^{*}}\right)^{k-1}$, so that

$$
\min _{t \in[0,1]} 1-F^{a}(t)=\min \left\{1,1-\frac{1}{\alpha}\left(e^{-\xi^{*}}+\xi^{*} e^{-\xi^{*}}+\frac{\xi^{*}}{k}\left(1-e^{-\xi^{*}}\right)-1\right)\right\} .
$$

Since $z \mapsto \frac{z\left(1-e^{-z}\right)}{1-e^{-z}-z e^{-z}}$ is increasing in $z$, we see that $\xi^{*} \geq \xi$ if and only if $\alpha \geq \alpha_{c}$ and we get

$$
\min _{t \in[0,1]} 1-F^{a}(t)=1-\mathbf{1}\left(\alpha \geq \alpha_{c}\right) \frac{1}{\alpha}\left(e^{-\xi^{*}}+\xi^{*} e^{-\xi^{*}}+\frac{\xi^{*}}{k}\left(1-e^{-\xi^{*}}\right)-1\right) .
$$




\section{Acknowledgement}

We would like to thank Andrea Montanari and Guilhem Semerjian for explaining us a key idea for the proof of Lemma 12, as well as Nikolaos Fountoulakis, David Gamarnik, James Martin and Johan Wästlund for interesting discussions.

The authors acknowledge the support of the French Agence Nationale de la Recherche (ANR) under reference ANR-11-JS02-005-01 (GAP project)

\section{References}

[1] D. Aldous and R. Lyons. Processes on unimodular random networks. Electronic Journal of Probability, 12:1454-1508, 2007.

[2] D. Aldous and J. M. Steele. The objective method: probabilistic combinatorial optimization and local weak convergence. In Probability on discrete structures, volume 110 of Encyclopaedia Math. Sci., pages 1-72. Springer, Berlin, 2004.

[3] D. J. Aldous and A. Bandyopadhyay. A survey of max-type recursive distributional equations. Ann. Appl. Probab., 15(2):1047-1110, 2005.

[4] J. Aronson, A. Frieze, and B. G. Pittel. Maximum matchings in sparse random graphs: Karp-Sipser revisited. Random Structures Algorithms, 12(2):111-177, 1998.

[5] M. Bayati, D. Gamarnik, and P. Tetali. Combinatorial approach to the interpolation method and scaling limits in sparse random graphs. In L. J. Schulman, editor, STOC, pages 105-114. ACM, 2010.

[6] M. Bayati and C. Nair. A rigorous proof of the cavity method for counting matchings. Proc. of the 44th Annual Allerton Conference on Communication, Control and Computing., 2006.

[7] I. Benjamini and O. Schramm. Recurrence of distributional limits of finite planar graphs. Electron. J. Probab., 6:no. 23, 13 pp. (electronic), 2001.

[8] I. Benjamini, O. Schramm, and A. Shapira. Every minor-closed property of sparse graphs is testable. In R. E. Ladner and C. Dwork, editors, STOC, pages 393-402. ACM, 2008.

[9] P. Billingsley. Convergence of probability measures. Wiley Series in Probability and Statistics: Probability and Statistics. John Wiley \& Sons Inc., New York, second edition, 1999. A Wiley-Interscience Publication.

[10] T. Bohman and A. Frieze. Karp-Sipser on random graphs with a fixed degree sequence. 2009.

[11] C. Bordenave and M. Lelarge. Resolvent of large random graphs. Random Structures Algorithms, 37(3):332-352, 2010.

[12] C. Bordenave, M. Lelarge, and J. Salez. The rank of diluted random graphs. Ann. Probab., 39(3):1097-1121, 2011. 
[13] C. Borgs, J. Chayes, J. Kahn, and L. Lovász. Left and right convergence of graphs with bounded degree. Arxiv preprint arXiv:1002.0115, 2010.

[14] Y.-B. Choe, J. G. Oxley, A. D. Sokal, and D. G. Wagner. Homogeneous multivariate polynomials with the half-plane property. Adv. in Appl. Math., 32(1-2):88-187, 2004. Special issue on the Tutte polynomial.

[15] M. Dietzfelbinger, A. Goerdt, M. Mitzenmacher, A. Montanari, R. Pagh, and M. Rink. Tight thresholds for cuckoo hashing via xorsat. In S. Abramsky, C. Gavoille, C. Kirchner, F. M. auf der Heide, and P. G. Spirakis, editors, ICALP (1), volume 6198 of Lecture Notes in Computer Science, pages 213-225. Springer, 2010.

[16] G. Elek. On limits of finite graphs. Combinatorica, 27(4):503-507, 2007.

[17] G. Elek and G. Lippner. Borel oracles. An analytical approach to constant-time algorithms. Proc. Amer. Math. Soc., 138(8):2939-2947, 2010.

[18] N. Fountoulakis and K. Panagiotou. Sharp load thresholds for cuckoo hashing. CoRR, abs/0910.5147, 2009.

[19] A. M. Frieze and P. Melsted. Maximum matchings in random bipartite graphs and the space utilization of cuckoo hashtables. CoRR, abs/0910.5535, 2009.

[20] C. D. Godsil. Matchings and walks in graphs. J. Graph Theory, 5(3):285-297, 1981.

[21] O. J. Heilmann and E. H. Lieb. Theory of monomer-dimer systems. Comm. Math. Phys., 25:190-232, 1972.

[22] R. Karp and M. Sipser. Maximum matchings in sparse random graphs. Proc. of the Twentysecond Annual Symposium on Foundations of Computer Science, IEEE:364-375, 1981.

[23] M. Lelarge. A new approach to the orientation of random hypergraphs. CoRR, abs/1201.5335, 2012.

[24] L. Lovász and M. D. Plummer. Matching theory. AMS Chelsea Publishing, Providence, RI, 2009. Corrected reprint of the 1986 original [MR0859549].

[25] R. Lyons. Asymptotic enumeration of spanning trees. Combin. Probab. Comput., 14(4):491$522,2005$.

[26] L. Zdeborová and M. Mézard. The number of matchings in random graph. Journal of Statistical Mechanics, 2006(5):P05003, 2006.

\section{Appendix : uniqueness and non-uniqueness at zero temperature}

Consider a sequence $\left(G_{n}=\left(V_{n}, E_{n}\right)\right)_{n \in \mathbb{N}}$ of finite graphs whose local weak limit under uniform rooting is a UGW tree $T$. Let $\phi(t)=\sum \pi_{n} t^{n}$ be the generating function of the degree distribution $\pi$ of $T$, and for $t \in[0,1]$ set

$$
F(t)=t \phi^{\prime}(1-t)+\phi(1-t)+\phi\left(1-\frac{\phi^{\prime}(1-t)}{\phi^{\prime}(1)}\right)-1
$$


From Theorem [16, we know that there is a.s. a unique solution to the local recursion at temperature $z=0$ on $T$ (correlation decay) if and only if the first local extremum of $F$ is a global maximum. In that case, the convergence

$$
\frac{\nu\left(G_{n}\right)}{\left|V_{n}\right|} \underset{n \rightarrow \infty}{\longrightarrow} \frac{1-\max _{t \in[0,1]} F(t)}{2}
$$

can be obtained by a fairly standard compactness-uniqueness argument, without any need for a detour through the positive temperature regime. As it is not hard to check, a sufficient condition for the first local extremum of $F$ to be a global maximum is that $\phi^{\prime \prime}$ is log-concave. This is in particular true in the Erdős-Rényi case, where $\phi(t)=\exp (c t-1),(c>0)$. The corresponding function $F$ is given in Figure 1 for various values of $c$.
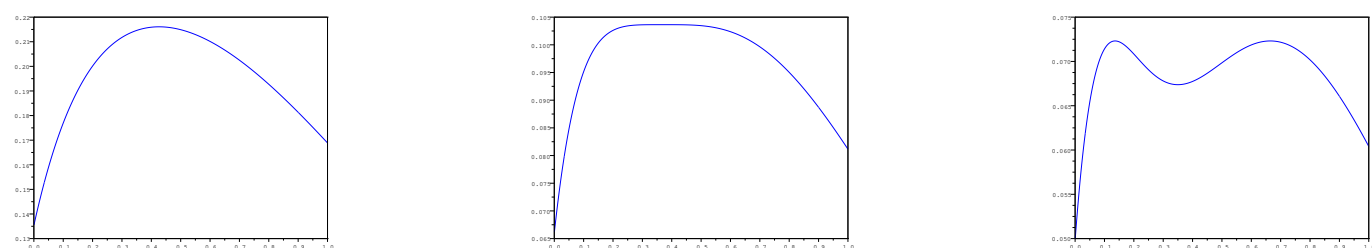

Figure 1: From left to right : plot of $F$ for $c=2, c=e$ and $c=3$.

However, there are simple examples of degree distributions $\pi$ for which the function $F$ has more than one historical record, implying the coexistence of multiple solutions to the local recursion (lack of correlation decay). In that case, a detour through the positive temperature regime is needed in order to establish the convergence (34). Here is a couple of examples.
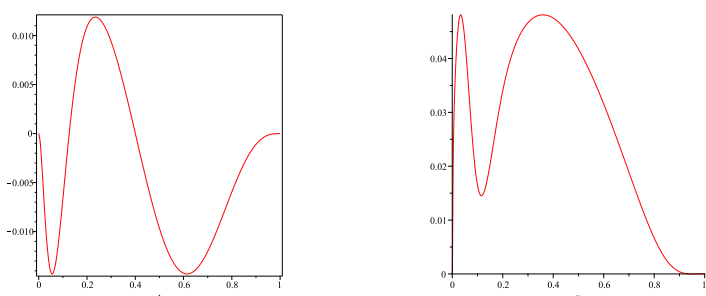

Figure 2: Plots of $F$ for $\phi(t)=\frac{3}{4} t^{3}+\frac{1}{4} t^{15}$ (left) and $\phi(t)=\frac{50}{101} t^{3}+\frac{50}{101} t^{20}+\frac{1}{101} t^{700}$ (right). 